\title{
Time-dependent craze zone growth at a crack tip in polymer solids
}

\author{
Wen-Bo Luo ${ }^{\mathrm{a}, *}$, Ting-Qing Yang ${ }^{\mathrm{b}}$, Xia-Yu Wang ${ }^{\mathrm{c}}$ \\ anstitute of Fundamental Mechanics and Material Engineering, Xiangtan University, Xiangtan, Hunan 411105, People's Republic of China \\ ${ }^{\mathrm{b}}$ Department of Mechanics, Huazhong University of Science and Technology, Wuhan 430074, People's Republic of China \\ 'School of Chemistry, Xiangtan University, Xiangtan, Hunan 411105, People's Republic of China
}

Received 15 September 2003; received in revised form 29 February 2004; accepted 5 March 2004

\begin{abstract}
By considering the polymer bulk as a linear viscoelastic body and the craze zone at crack tip as a nonlinear damage zone, the control equation for craze zone growth has been derived. It is shown that for a time-independent craze-zone stress, the craze zone would grow only if the crack-tip stress intensity factor is changed. If the crack-tip stress intensity factor remains constant during loading, the growth rate of the craze zone length will be interrelated to the crack-tip stress, the craze zone length and the rate of change of the craze-zone stress. If both the craze-zone stress and the crack-tip stress intensity factor are time-independent, the craze zone length will be constant during the crack growth, which is the case of self-similar crack growth. Moreover, a new stress distribution model in craze zone is presented based on the constructed damage evolution law, and the lengthening and thickening of the craze zone at the crack tip are also formulated. The numerical calculations from the proposed model agree well with the published experimental data.
\end{abstract}

(C) 2004 Elsevier Ltd. All rights reserved.

Keywords: Crazing; Damage zone; Crack

\section{Introduction}

Polymers are replacing metallic materials in many loadbearing applications. Strength and toughness are two of the most important mechanical properties of structural materials, such as polymers. Therefore, strengthening and toughening of polymers have been the focus of research attention since 1980s, which are based on the understanding of the deformation and failure mechanisms. As to glassy polymers, crazing controls much of the macroscopic response of the material. When crazing occurs under wellcontrolled conditions, as in high impact polystyrene (HIPS) blends, it provides a mechanism of inelastic deformation improving the material toughness, because considerable energy dissipates in the processes of craze initiation, craze growth and craze fibril breakdown, there is not so much energy to drive the crack propagation, thus crazing constrains the crack growth to a certain extent. On the other hand crazes are usually the precursor of cracks and, ultimately, gross failure. Despite considerable advances in the understanding of crazing in polymers, there are many

\footnotetext{
* Corresponding author. Tel.: + 86-7328293240; fax: + 86-7328293659.

E-mail address: luowenbo@xtu.edu.cn (W.B. Luo).
}

issues of considerable interest in many important interrelated processes such as craze initiation, craze tip advance, craze thickening and craze breakdown that are still unresolved [1,2]. Among those processes, craze tip advancing and craze thickening are especially crucial since they are responsible for the major part of energy dissipation during crazing and thus directly influence the material toughness. In this paper, by considering the polymer bulk as a linear viscoelastic body and the craze zone at the crack tip as a nonlinear damage zone, the control equation for craze zone growth is derived. Moreover, a new simple craze-stress distribution is presented based on the constructed damage evolution law, and the lengthening and thickening of the craze zone at the crack tip are also formulated. Numerical calculations from the proposed model agree well with the published experimental data.

\section{Craze zone growth at crack tip}

Under certain thermo-mechanical conditions, crazing may occur on the surfaces, or inside the bulk of polymers. It may also form at the crack tips. Fig. 1 shows the SEM micrographs of the surface and inner craze in polypropylene 

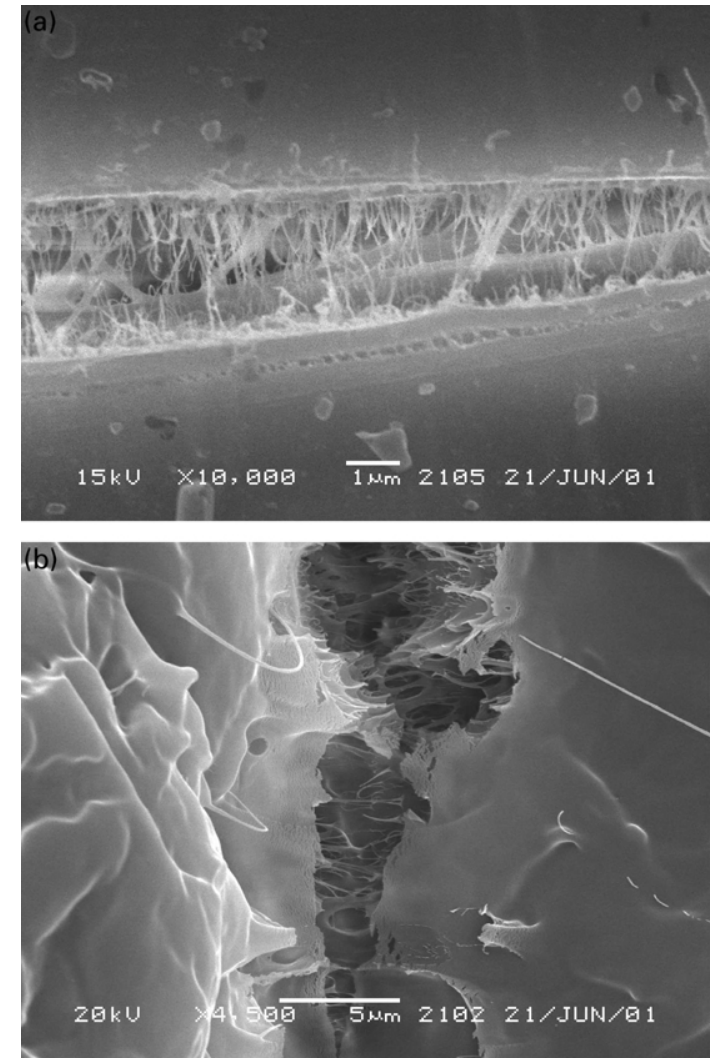

Fig. 1. (a) SEM micrograph of surface craze in PP; (b) SEM micrograph of inner craze in PP.

(PP) specimens. From which it can be seen that craze consists of voids and highly orientational fibrils, which connect each other to form a network structure. The craze can usually contain voids with volume fraction of 50-80 percent. The fibril diameter and spacing are only a few or tens of nanometers, which are strongly dependent on the molecular structure of polymers, environment temperature, loading rate and stress levels.

The craze grows in two different ways [2]: by craze tip advance, an expansion of the craze periphery generating more fibrils, and by craze width growth, a normal separation of the two craze-interfaces behind the craze tip. The general mechanism of craze tip advance has been well known to be the Taylor meniscus instability process; whereas the mechanism of craze thickening is till open.

\subsection{Control equations for craze zone growth at crack tip}

Polymers are usually viscoelastic in mechanical behavior. Their deformation and failure processes are time-, temperature- and rate-dependent. The effects of viscoelasticity on crack growth have been treated by a number of workers, among them are Williams [3], Knauss [4,5], Schapery [6], and McCartney [7]. To describe the work of these authors is clearly beyond the scope of this paper. Below we will discuss the treatment of Schapery [6] since it is typical and will be used in this paper.
Schapery's approach treats the crack growth problem in viscoelastic media by applying elasticity-viscoelasticity correspondence principle to the Barenblatt theory for an elastic body. Schapery's results pertain to the initiation of crack growth and continuous steady state growth of a fully developed crack-tip process zone. Care must be taken when the boundary conditions are time dependent, in this case an extended correspondence principle proposed by Graham [8] can be applied under certain conditions. Following Barenblatt, Schapery considers a failure zone exists at the crack tip. This may represent stretched bonds as in the original Barenblatt concept, or any kind of materials, including crazes we shall investigate below, provided that the stress distribution in this failure zone is time independent.

Following Schapery's approach, in this paper we investigate the time dependent growth of the craze zone at a crack tip in linearly viscoelastic media. For our purpose, we shall consider the failure zone in Schapery's approach to be the craze zone. Moreover, the craze zone is treated as a nonlinear damage zone, and in which it is not required to have a uniform stress distribution.

Consider an infinite linearly viscoelastic plate with a crack length $2 a(t)$ under a remotely tensile stress $\sigma_{\infty}(t)$ (see Fig. 2). The crack lies on the $x O z$ coordinate plane. The crack center is located at the origin of the coordinate system. The length of the crack-tip craze zone is $l(t)$ and the distance from the craze tip to the crack center is $c(t)$.

Take the cracked body excluding the crack-tip craze zone under consideration. In this case, the crack is virtually extended from the actual crack tip at $x=a(t)$ by the craze zone length $l(t)$. Within this zone, the crack surfaces are loaded by the cohesive stress $\sigma_{\mathrm{c}}(x, t)$.

With the small craze zone at crack tip in mind, the virtual crack tip (actually the craze zone tip) stress intensity factor $K_{\text {tip }}^{\mathrm{CZ}}$ can be superposed from the stress intensity factor $K_{\mathrm{I}}$ induced by the remote stress $\sigma_{\infty}$ and the stress intensity factor $K_{\mathrm{I}}^{\prime}$ induced by the cohesive stress $\sigma_{\mathrm{c}}(x, t)$ in the craze zone.

$K_{\mathrm{I}}=\sigma_{\infty} \sqrt{\pi c}$

$K_{\mathrm{I}}^{\prime}=-2 \sqrt{\frac{c}{\pi}} \int_{a}^{c} \frac{\sigma_{\mathrm{c}}(x, t)}{\sqrt{c^{2}-x^{2}}} \mathrm{~d} x$

A local abscissa $\xi$, coaxial with the crack and with the origin at the craze zone tip is introduced, as shown in Fig. 2. We can easily find the interrelation between $\xi$ and $x$ by $\xi=c-x$. For the case of small craze zone at crack tip, $\xi / c \ll 1$, then Eq. (2) can be rewritten as

$$
\begin{aligned}
K_{\mathrm{I}}^{\prime} & =-\sqrt{\frac{2}{\pi}} \int_{0}^{l} \frac{\sigma_{\mathrm{c}}(\xi, t)}{\sqrt{\xi}} \sqrt{\frac{1}{1-\xi / 2 c}} \mathrm{~d} \xi \\
& \approx-\sqrt{\frac{2}{\pi}} \int_{0}^{l} \frac{\sigma_{\mathrm{c}}(\xi, t)}{\sqrt{\xi}} \mathrm{d} \xi
\end{aligned}
$$






Fig. 2. The crack tip model with the craze zone.

thus

$K_{\text {tip }}^{\mathrm{CZ}}=K_{\mathrm{I}}-\sqrt{\frac{2}{\pi}} \int_{0}^{l} \frac{\sigma_{\mathrm{c}}(\xi, t)}{\sqrt{\xi}} \mathrm{d} \xi$

To eliminate the stress singularity at the virtual crack tip, $K_{\text {tip }}^{\mathrm{CZ}}$ should be equal to zero. So

$K_{\mathrm{I}}=\sqrt{\frac{2}{\pi}} \int_{0}^{l}\left[\sigma_{\mathrm{c}}(\xi, t) / \sqrt{\xi}\right] \mathrm{d} \xi$

This equation gives a relation among the cohesive stress in the craze zone $\sigma_{\mathrm{c}}(\xi, t)$, the craze zone length $l(t)$ and the stress intensity factor $K_{\mathrm{I}}(t)$ due to remote stress $\sigma_{\infty}$. Differentiating this equation with respect to time, we obtain the control equation for crack-tip craze zone growth:

$\frac{\sigma_{\mathrm{c}}(l, t)}{\sqrt{l}} \frac{\mathrm{d} l}{\mathrm{~d} t}=\sqrt{\frac{\pi}{2}} \frac{\mathrm{d} K_{\mathrm{I}}}{\mathrm{d} t}-\int_{0}^{l} \frac{\partial \sigma_{\mathrm{c}}(\xi, t)}{\partial t} \frac{\mathrm{d} \xi}{\sqrt{\xi}}$

It can be seen that for a time-independent craze-zone stress, the craze zone grows only if the crack-tip stress intensity factor $K_{\mathrm{I}}$ is changed. If $K_{\mathrm{I}}$ remains constant during loading, the growth rate of the craze zone length will be interrelated to the crack-tip stress $\sigma_{\mathrm{c}}(\xi=l, t)$, the craze zone length and the rate of change of the craze-zone stress. If both the craze-zone stress and the crack-tip stress intensity factor are time-independent, the craze zone length will be constant during crack growth, which is the case of self-similar crack growth.

For simplicity, we consider the craze zone growth behavior at a quasi-static crack tip. In this case, $\dot{a}=0, \dot{l} \neq 0$.. It can be seen from Eq. (6) that in order to get the craze zone growth law, the external load and the distribution of craze-zone stress and its variation with time must be known. Usually the analytical solution of this problem is too difficult to be gotten, so some simplification hypotheses are made.

For the case of constant external load, with the expression of $c=a+l$ in mind, we can get the following equation by substituting Eq. (1) into Eq. (6):

$\left(\frac{\sigma_{\mathrm{c}}(l, t)}{\sqrt{l}}-\frac{\pi \sigma_{\infty}}{2 \sqrt{2(a+l)}}\right) \frac{\mathrm{d} l}{\mathrm{~d} t}=-\int_{0}^{l} \frac{\partial \sigma_{\mathrm{c}}(\xi, t)}{\partial t} \frac{\mathrm{d} \xi}{\sqrt{\xi}}$

Let $\eta=\xi / l$, which normalizes the craze zone length, and then the above equation can be rewritten as:

$$
\begin{gathered}
\frac{\sigma_{\mathrm{c}}(l, t)}{l}\left(1-\frac{\pi}{2 \sqrt{2}} \frac{\sigma_{\infty}}{\sigma_{\mathrm{c}}(l, t)} \sqrt{\frac{l}{a+l}}\right) \frac{\mathrm{d} l}{\mathrm{~d} t} \\
=-\int_{0}^{1} \frac{\partial \sigma_{\mathrm{c}}(\eta l, t)}{\partial t} \frac{\mathrm{d} \eta}{\sqrt{\eta}}
\end{gathered}
$$

For the case of constant crack-tip stress intensity factor, Eq. (6) can be simplified as:

$\frac{\sigma_{\mathrm{c}}(l, t)}{\sqrt{l}} \frac{\mathrm{d} l}{\mathrm{~d} t}=-\int_{0}^{l} \frac{\partial \sigma_{\mathrm{c}}(\xi, t)}{\partial t} \frac{\mathrm{d} \xi}{\sqrt{\xi}}$

By the substitution $\eta=\xi / l$, the above equation can be transformed into:

$\frac{\sigma_{\mathrm{c}}(l, t)}{l} \frac{\mathrm{d} l}{\mathrm{~d} t}=-\int_{0}^{1} \frac{\partial \sigma_{\mathrm{c}}(\eta l, t)}{\partial t} \frac{\mathrm{d} \eta}{\sqrt{\eta}}$

It can be seen from Eq. (10) that the craze-zone stress will decrease with time during the craze zone growth. This prediction is consistent with the published test data [9]. The calculations provided by Yang and co-workers [10] also showed that the average stress in the craze zone decreases with time.

Comparing Eq. (8) with Eq. (10), it can be found that Eq. (8) reduces to Eq. (10) if

$\frac{\pi \sigma_{\infty}}{2 \sqrt{2} \sigma_{\mathrm{c}}(l, t)} \sqrt{\frac{l}{a+l}} \ll 1$,

which is true when $l / a \ll 1$ and since $\sigma_{\mathrm{c}}(l, t)$ is not expected to be a great deal smaller than the remote stress $\sigma_{\infty}$. So, we 
only consider the case of constant stress intensity factor infra for computational conveniences.

\subsection{Stress distribution in craze zone at crack tip}

For a sharp crack, in a linearly viscoelastic body, with no damage or yielding zone at its tips, there exists a singularity of $-1 / 2$ for the crack-tip stresses [6]. However, for real cases, the damage or yielding occurs to form a damage or yielding zone at the crack tips, which is called craze zone in this paper, and plays a relaxation role on the crack-tip stresses, so the crack-tip stresses are bounded. We can imagine that the craze zone comes into existence at a quasi-static crack tip in polymers when the crack-tip stress reaches to the craze initiation stress, $\sigma_{\text {ini }}$. The craze fibrils get weaker and weaker under the craze-zone stresses, their load-bearing capabilities will decrease progressively. When the fibrils at crack tips cannot bear load anymore, the crack tip begins to advance. So during the crack growth, the crack-tip stress $\sigma_{\mathrm{c}}(\xi=l)$ would be zero. From the above description, we can get a picture of the stress distribution in the craze zone and its variation with time before the initiation of crack growth, as shown in Fig. 3. It can be seen that during the craze growth, the stress at craze-zone tip remains a constant value of $\sigma_{\text {ini }}$, the craze initiation stress. The crack-tip stress $\sigma_{\text {tip }}=\sigma_{\mathrm{c}}(\xi=l, t)$, however, decreases with time till to zero, at this instant the crack tip starts to advance. Thus we can suppose that the stress distribution in the craze zone and its variation with time has the form as:

$\sigma_{\mathrm{c}}(\xi, t)=\sigma_{\mathrm{ini}}+\left[\sigma_{\mathrm{tip}}(t)-\sigma_{\mathrm{ini}}\right]\left[\frac{\xi}{l(t)}\right]^{m}$

where the crack-tip stress $\sigma_{\text {tip }}(t)$ has the following form:

$\sigma_{\text {tip }}(t)=\sigma_{\mathrm{c}}(l, t)=\sigma_{\text {ini }}\left[1-\left(\frac{t}{t_{\mathrm{i}}}\right)^{n}\right]$

in which, $t_{\mathrm{i}}$ is the time span from craze initiation to crack tip advance, which may depend on the material strength and the external load condition. For brittle polymers, $t_{\mathrm{i}}$ can be considered to be the material's lifetime. The parameters $m$ and $n$ in Eqs. (11) and (12) are material constants. They are positive and less than 1 , and they are introduced to describe

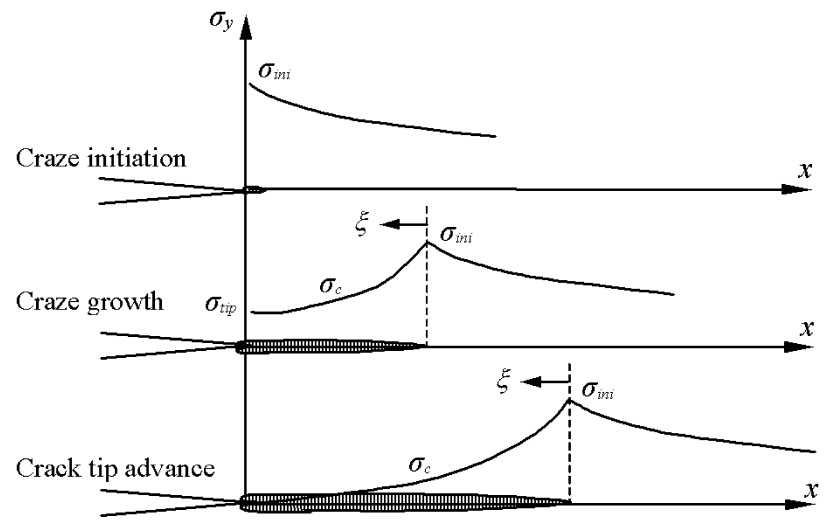

Fig. 3. Craze stress model of the craze zone at a crack tip. the loss of mechanical properties of the material in the craze zone due to the deformation and the load-bearing time. The smaller $m$ and $n$ are, the less $\sigma_{\mathrm{c}}(\xi, t)$ and $\sigma_{\text {tip }}(t)$ would be, that is, the more the load-bearing capacity of the material in craze zone would decrease, leading to damage softening. As to the physical meaning of these two parameters, it is easy to see form the analysis below that $m$ and $n$ determine the damage evolution law of the material in crack-tip craze zone. Substituting Eq. (12) into Eq. (11) yields

$\sigma_{\mathrm{c}}(\xi, t)=\sigma_{\text {ini }}\left[1-\left(\frac{\xi}{l(t)}\right)^{m}\left(\frac{t}{t_{\mathrm{i}}}\right)^{n}\right]$

Let:

$D(\xi, t)=\left(\frac{\xi}{l(t)}\right)^{m}\left(\frac{t}{t_{\mathrm{i}}}\right)^{n}$

then $\sigma_{\mathrm{c}}(\xi, t)=[1-D(\xi, t)] \sigma_{\text {ini }}$. It is shown that $D(\xi, t)$ can be considered as a damage variable to describe the crazing damage. Eq. (14) is the damage evolution law. From which it is seen again that the smaller $m$ and $n$ are, the greater the damage would be.

From Eqs. (13) and (14), the crazing damage evolution and the stress distribution and its variation with time can be obtained for the given material constants. Obviously, $m$ and $n$ have various values for different materials. Figs. 4 and 5 show the examples for $m=0.55$ and $n=0.2$, which indicate the case of PMMA [11].

\subsection{Craze zone lengthening and thickening at crack tip}

Craze zone grows in two ways: craze tip advance along the crack extension direction; and craze zone thickening. For the case of constant stress intensity factor, substituting Eq. (13) into Eq. (5), we can get the control equation for the craze zone lengthening:

$l(t)=\frac{\pi}{8}\left(\frac{K_{\mathrm{I}}}{\sigma_{\text {ini }}}\right)^{2}\left[1-\frac{1}{2 m+1}\left(\frac{t}{t_{\mathrm{i}}}\right)^{n}\right]^{-2}$



Fig. 4. Distribution and evolution of the crazing damage. 


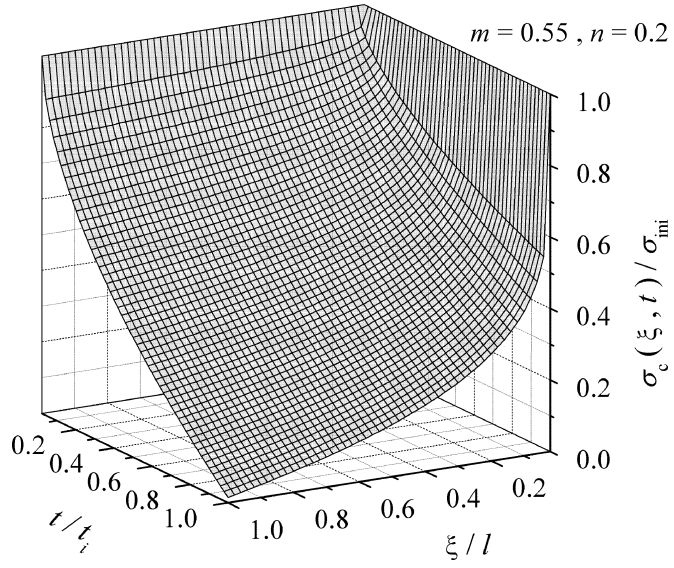

Fig. 5. Craze stress distribution and variation with time.

It can be seen that the length of craze zone increases with time. At $t=0^{+}$the initial length of the craze zone is

$\frac{\pi}{8}\left(K_{\mathrm{I}} / \sigma_{\text {ini }}\right)^{2}$

which is the expected result for a constant stress over the craze zone as in Dugdale model for small scale yielding.

As to the initiation of crack growth in linearly viscoelastic medium, Schapery [6] proposed a model to calculate the viscoelastic opening displacement, $2 v(\xi, t)$, in crack-tip failure zone, which is termed as the crack-tip craze zone in this paper.

$$
\begin{aligned}
2 v(\xi, t)= & -\frac{16}{3 \pi} H(\xi) \int_{t_{1}}^{t} C(t-\tau) \frac{\partial}{\partial \tau} \\
& \times\left\{\xi^{\frac{3}{2}} \int_{0}^{1} \frac{\partial \sigma_{\mathrm{c}}(\zeta, \tau)}{\partial \zeta} \frac{1}{\sqrt{\zeta}} \mathrm{d} \zeta\right\} \mathrm{d} \tau
\end{aligned}
$$

where

$C(t)=\left\{\begin{array}{ll}J(t), & \text { plane stress } \\ \left(1-v^{2}\right) J(t), & \text { plane strain }\end{array}\right.$,

and $J(t)$ denotes the creep compliance of the bulk material, $t$ is time, $t_{1}$ is the time span for crack tip to reach the point with fixed $x$ along the crack extension direction, and $\tau$ is the integral variable. Substituting Eq. (13) into Eq. (16) leads to

$2 v(\xi, t)=\frac{16 m \sigma_{\mathrm{ini}}}{3 \pi(m-1 / 2) t_{\mathrm{i}}^{n}} \int_{t_{1}}^{t} C(t-\tau) \frac{\partial}{\partial \tau}\left[\xi^{3 / 2} \tau^{n} / \sqrt{l(t)}\right] \mathrm{d} \tau$

From which, it is seen that $m>0.5$ for the constraint of positive opening displacement. Note that the time integral in the above equation is only conducted for points with fixed $x$, not for points with fixed $\xi$. With $\xi=a-x+l(t)$ in mind, the above equation can be rewritten as

$$
\begin{aligned}
& 2 v(x, l, t)=\frac{16 m \sigma_{\text {ini }}}{3 \pi(m-1 / 2) t_{\mathrm{i}}^{n}} \int_{\mathrm{t}_{1}}^{\mathrm{t}} C(t-\tau) \\
& \quad \times\left\{\left[\frac{3}{2} \tau^{n}\left(\frac{a-x+l(\tau)}{l(\tau)}\right)-\frac{1}{2} \tau^{n}\left(\frac{a-x+l(\tau)}{l(\tau)}\right)^{3 / 2}\right] \dot{l}(\tau)\right. \\
& \left.\quad+n l(\tau) \tau^{n-1}\left(\frac{a-x+l(\tau)}{l(\tau)}\right)^{3 / 2}\right\} \mathrm{d} \tau, \quad x \in[a, c]
\end{aligned}
$$

As we know, craze consists of holes and highly oriented fibrils. The craze widening mechanism is still open; though there are already two possible explanations, i.e. creep thickening mechanism and surface drawing thickening mechanism [1]. The two mechanisms are not mutually exclusive and do coexist for particular conditions such as environmental crazing. However, in the case of air crazes, the currently accepted explanation to craze thickening is due to Kramer and Berger [2] who have suggested that the fibrillation in craze zone occurs by a local drawing process of new polymers from an 'active zone' near the craze/bulk interface into the fibrils. Suppose that $2 w_{0}(x, l, t)$ is the primordial thickness of the crazed zone, that is the thickness of the layer of bulk which fibrillates to form a craze, and $2 w(x, l, t)$ the craze zone thickness (see Fig. 6), then the craze zone opening displacement $2 v(x, l, t)$ can be expressed by

$2 v(x, l, t)=2 w(x, l, t)-2 w_{0}(x, l, t), \quad x \in[a, c]$,

By introducing the craze extension ratio, which can be defined as the ratio of the craze zone thickness to its primordial thickness, i.e. $\lambda(x, l, t)=2 w(x, l, t) / 2 w_{0}(x, l, t)$, we can easily get Eq. (20) from Eq. (19).

$2 w(x, l, t)=\left(\frac{\lambda}{\lambda-1}\right) 2 v(x, l, t), x \in[a, c]$

For the point with $x=a$, i.e. the crack tip, we can obtain the maximum thickness of the craze zone, $\delta$, from Eqs. (18) and (20). For a stationary crack under a constant stress intensive factor, the time $t_{1}$ for craze zone tip to reach the fixed point with $x=a$ is 0 . Namely, the craze zone can be formed at the crack tip at the moment when the external load is applied to

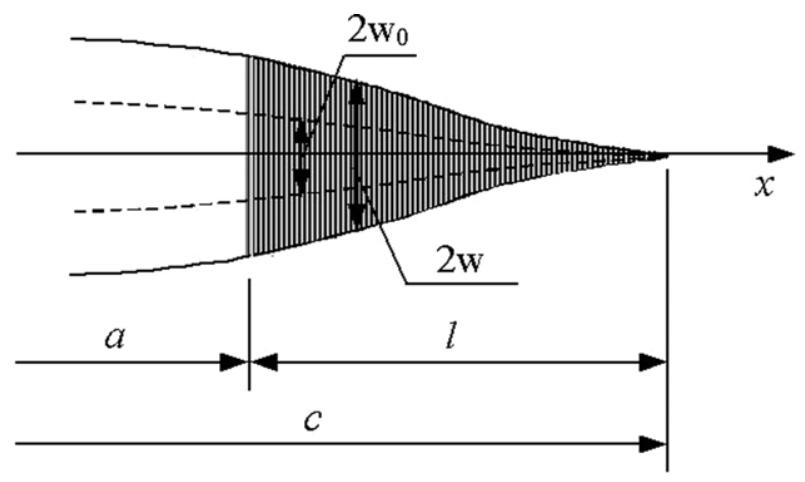

Fig. 6. Geometry of the craze zone. 
the cracked body. So

$$
\begin{aligned}
\delta= & \frac{16 m \sigma_{\text {ini }}}{3 \pi(m-1 / 2) t_{\mathrm{i}}^{n}}\left(\frac{\lambda}{\lambda-1}\right) \int_{0}^{t} C(t-\tau) \\
& \times\left[\tau^{n} \dot{l}(\tau)+n l(\tau) \tau^{n-1}\right] \mathrm{d} \tau
\end{aligned}
$$

At the moment of $t=0^{+}$, the craze zone length can be expressed by

$l_{0}=\frac{\pi}{8}\left(\frac{K_{\mathrm{I}}}{\sigma_{\mathrm{ini}}}\right)^{2}$,

and the craze zone width at the crack tip $\delta_{0}=K_{\mathrm{I}}^{2} / E \sigma_{\text {ini }}$, where $E$ is the transient elastic modulus of the bulk material. Thus, from Eq. (21) we get

$$
\begin{aligned}
\delta= & \frac{16 m \sigma_{\text {ini }}}{3 \pi(m-1 / 2) t_{\mathrm{i}}^{n}}\left(\frac{\lambda}{\lambda-1}\right) \int_{0^{+}}^{t} C(t-\tau) \\
& \times\left[\tau^{n} \dot{l}(\tau)+n l(\tau) \tau^{n-1}\right] \mathrm{d} \tau+\delta_{0}
\end{aligned}
$$

\section{Numerical example}

In numerical calculations, we take PMMA, a typical amorphous polymer, as an example. Let $K_{\mathrm{I}}=0.6 \mathrm{MPa} \sqrt{m}$ (i.e. $19 \mathrm{~N} / \mathrm{mm}^{3 / 2}$ ), $t_{\mathrm{i}}=8 \times 10^{6} \mathrm{~s}$, the craze initiation stress $\sigma_{\text {ini }}=70 \mathrm{MPa}, m=0.55$ and $n=0.2$ [11], the calculated craze zone lengths are shown in Fig. 7. For calculation of the craze zone width, let $E=3.5 \mathrm{GPa}$ and $\lambda=2$ [12], then we get $\delta_{0}=1.47 \times 10^{-6} \mathrm{~m}$. Suppose the creep compliance of the bulk material has the form as $C(t)=C_{1}+C_{2}[1-$ $\left.\exp \left(-t / \tau_{0}\right)\right]$, where $C_{1}=1 / E, C_{2}=4 \times 10^{-4} \mathrm{MPa}^{-1}, \tau_{0}=$ $10^{10} \mathrm{~s}$. The calculated maximum widths of the craze zone from Eq. (22) are shown in Fig. 8 and compared with the published test data. It can be seen that the numerical results agree well with the published experimental data by Döll [9].

\section{Concluding remarks}

The crack-tip crazing damage and its evolution in

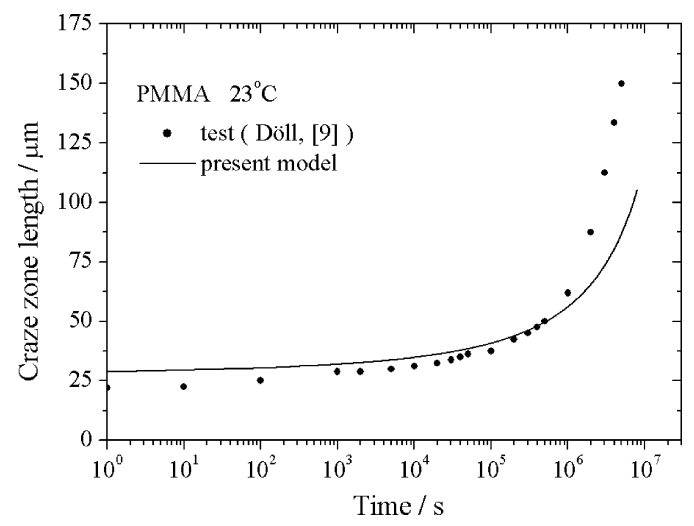

Fig. 7. Craze zone length variation with time.

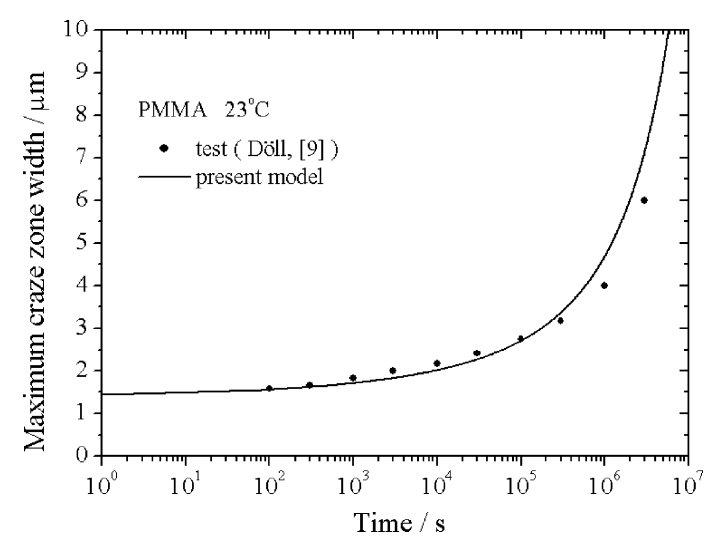

Fig. 8. Maximum craze zone width vs time.

polymers are considered in this paper. The control equation for the growth of crack-tip craze zone in viscoelastic medium has been derived. It is shown that for a timeindependent craze-zone stress, the craze zone would grow only if the crack-tip stress intensity factor is changed. If the crack-tip stress intensity factor remains constant during loading, the growth rate of the craze zone length will be interrelated to the crack-tip stress, the craze zone length and the rate of change of the craze-zone stress. If both the crazezone stress and the crack-tip stress intensity factor are timeindependent, the craze zone length will be constant during crack growth, which is the case of self-similar crack growth. By considering the polymer bulk as a linear viscoelastic body and the craze zone at crack tips as a nonlinear damage zone, a new model for the craze-zone stress distribution and its variation with time is presented based on the constructed crazing damage evolution law. Furthermore, the variation of the length and width of the crack-tip craze zone with time are obtained. The numerical calculations for PMMA agree well with the published experimental data.

\section{Acknowledgements}

This work is supported by the National Natural Science Foundation of China $(10172037,20204012)$ and Hunan Provincial Education Department (02B005).

\section{References}

[1] Narisawa I, Yee AF. In: Crazing and fracture of polymers. Thomas EL, editor. Structure and properties of polymers, materials science and technology, a comprehensive treatment, vol. 12. Weinheim: VCH; 1993. p. 698-765.

[2] Kramer EJ, Berger LL. Fundamental processes of craze growth and fracture. In: Kausch HH, editor. Crazing in polymers, advances in polymer science 91/92. Berlin: Springer; 1990. p. 1-68.

[3] Williams JG. Fracture mechanics of polymers. Chichester: Ellis Horwood Limited Publishers; 1980.

[4] Knauss WG, Losi GU. J Appl Mech 1993;60:793-801.

[5] Knauss WG. J Engng Mater Tech 1993;115:262-7.

[6] Schapery RA. Int J Fracture 1975;11:141-59. 
[7] McCartney LN. Int J Fracture 1988;37:279-301.

[8] Graham GAC. Q Appl Math 1968;26:167-74.

[9] Doll W. Polym Engng Sci 1984;24:798-808.

[10] Yang TQ, An QL, Luo WB. Polym Engng Sci 2001;41:1171-6.

[11] Luo Wenbo. Studies on deformation-induced heat effect, nonlinear viscoelastic behavior and crazing in polymers. PhD Dissertation, Huazhong University of Science and Technology, Wuhan, China; 2001.

[12] Lauterwesser BD, Kramer EJ. Philos Mag 1979;A39:469-95. 